\title{
A Methodology and Design Process for System Generated User Interruption based on Context, Preferences, and Situation Awareness
}

\author{
Ashish Godbole and Waleed W. Smari \\ Department of Electrical and Computer Engineering \\ University of Dayton \\ 300 College Park, Dayton, Ohio 45469-0226 \\ \{godbolas, Waleed.Smari@notes.udayton.edu\}
}

\begin{abstract}
Over the past few decades, electronic devices and computing systems have evolved from being rigid, complex and inflexible, to becoming highly dynamic, and responsive. While these properties have the potential to make them user friendly and human centric, they also bring about inherent drawbacks, such as user interruptions caused by a variety of system generated events. In general, interruptions cannot be eliminated and hence need to be handled since they are essential for activities such as interaction, event notification, automation, and so on. This paper discusses the fundamentals of system generated interruption and presents a comprehensive taxonomy that incorporates past and current works on interruption handling techniques and solutions. The paper also proposes a methodology and design process for the developing of interruption aware systems that are based on the use of knowledge gathered from users' preferences, surroundings and situations via information elicitation. These incorporate user based design techniques, learning mechanisms, and preference elicitation methods in order to develop models that accurately take context, user's preferences/profiles and situations into account while designing for interruption awareness.
\end{abstract}

Keywords: Context Aware Systems, Interruption Handling, Usability Engineering, Information Elicitation, User Profiling and Preference.

\section{Introduction}

With the growing variety of scenarios, environments, and end users, system designers have begun to look at incorporating features to provide human centric capabilities in their designs. This is being done by focusing on both human factors (psychology, sociology, culture, etc.) and engineering disciplines (context awareness, AI, HCI, etc.). While these areas offer great promise, they are not without drawbacks and concerns such as interruptions, privacy, security, control, and so on. This paper focuses on addressing one such drawback inherent in today's human centric systems, namely the problem of user interruptions caused by system generated events, notifications, or errors. While this problem may not be very serious today, it could become so in the near future with the growing popularity of pervasive and context aware systems, intelligent phones, smart homes and other environments. This problem is important because interruptions are necessary for a variety of reasons; maybe to notify the user of impending tasks, warnings and errors, or elicit information from the user to continue current activities, or even periodically update user with the state of activities in progress. Since such interruptions cannot be eliminated, they need to be handled in a manner that does not affect system services or human system interaction, but at the same time reduces their deleterious effects on user performance and activities. We focus on the fundamentals of system generated interruption and discuss in detail existing solutions for handling this problem, with the help of a comprehensive taxonomy. We outline a methodology for designing these systems and propose incorporating user preferences and other relevant information in the system design process to assist designers in reducing interruptions and their impact. Implementation and examples of our approach are discussed briefly in this paper but are the focus of another forthcoming work [59].

The paper is organized as follows. Section 2 presents a background of system generated interruptions along with their types, properties, causes, and effects. Section 3 introduces a taxonomy of existing solutions for handling interruptions. Section 4 outlines our approach for interruption aware systems design while Section 5 presents a design process that incorporates our approach for developing interruption aware systems. Section 6 concludes the paper with a summary and discussion on future work and directions in interruption awareness.

\section{Interruptions: Background, Properties, and Effects}

The following definition has been considered as a general unified definition for interruption. It covers interruption caused to humans by other humans and interruption caused to humans by systems or devices. 
“... Interruption is the process of coordinating abrupt change in people's activities. ... Postulate 1: This abrupt change involves one or more of a person's modes of activity: (1) cognition (2) perception, or (3) physical action." [41]

Based on the above definition, the following working definition of system generated interruption is provided:

"A break in the continuity or uniformity of a user's activity, focus, or cognition, caused by system generated events during human-system interaction."

There is considerable work on the general effects of interruption, both human and system generated, and their causes and properties [18, 28, 39, 49]. Research in user interruption handling, resolution and awareness, on the other hand, is quite recent and not extensive [28, 38, 52]. In all, as we shall see in the next sections, most efforts concentrate on rigid implementations and static rules that are not compatible across domains. Figure 1 illustrates the characteristics, types and causes of system generated user interruption. System generated interruptions can be caused by a variety of factors, as indicated by many researchers [13, 14, 21, 28, 39, 53].

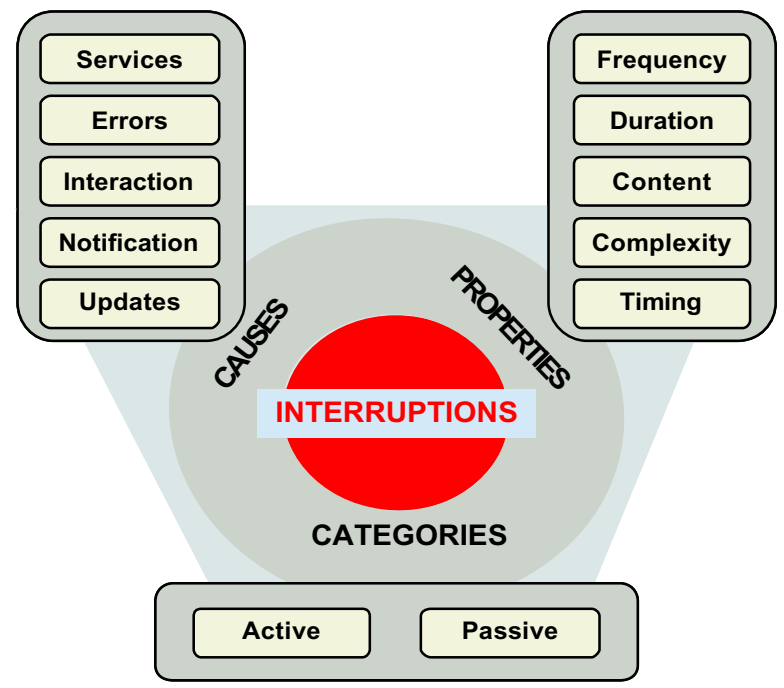

Figure 1. The Basic Fundamentals of System Generated User Interruption

Given the diversity of systems today, some of these factors are the services they provide, errors that may occur, notifications, updates, and user-system or user-user interaction, etc. The Figure also illustrates the main characteristics of interruptions, namely, frequency, duration, content, complexity, and timing. Essentially, these properties identify how often they occur in a system, how long they last, what is their purpose, and how much work the user will have to do to resolve them. Although there may be many ways of classifying interruptions, we prefer to categorize them based on the user's perceptibility of an interruption. In this regard, active interruptions are interruptions that are highly perceptible by the user when compared to passive interruptions which can be thought of as peripheral in nature and which do not directly attract the user's attention.

Interruptions are prevalent in many of today's applications and scenarios: control stations, airplane cockpits, collaborative systems, cell phones, and so on. Such systems are highly dynamic, responsive, multimodal, and pervasive. These features make them prone to frequent, untimely, sudden, or even erroneous interruptions. The effects of such interruptions can range from mildly annoying to catastrophic $[8,33,55]$. There have been many experimental and field studies performed in an attempt to measure the benefits and drawbacks of interruptions. Some measured the cost of interruptions in terms of recovery time [21, 53], task execution [2], accuracy of post-interruption tasks [30], and even evaluation of learned models [26, 27]. Others focused on the effects in terms of physical and mental stress [32]. Generally, there is an overall agreement that the hindrances caused due to interruptions largely outnumber their benefits. Based on the literature, the following is a summary of the effects of interruption:

- Delays: loss of time. Work schedules and activities are based on interruption frequency, complexity \& longevity.

- Errors: manual or random. May be mildly annoying or catastrophic in nature. Can cause additional hindrances such as delays, loss of continuity, etc.

- Increased Cognitive Load: occurs when user has to switch from ongoing activity to handle the interruption.

- Loss of Continuity: when user is performing multiple tasks, or when the interruption requires him to be away from his original task for considerable period of time. Delays and increased cognitive workload may result.

- Loss of Focus and Concentration: untimely or sudden interruptions, use of active interruption modalities. Common examples are phone ringers, alarms, and so on.

- Socio-psychological Effects: annoyance, anger, or frustration, etc. May lead to other factors such as errors, delays, loss of focus and concentration, etc.

- Cost: evaluated based on factors such as loss of time, work, human errors, equipment cost, etc. May be measured by quantifying some of these effects.

To summarize, interruptions are essential. They cannot be eliminated and their effects may range from mildly annoying to catastrophic. In the next section we will look at existing interruption handling techniques.

\section{Interruption Handling}

Over the past two decades, there have been some encouraging efforts to tackle the issue of handling user interruptions. Figure 2 illustrates a taxonomy representing various solutions for handling system generated interruptions. These efforts can be categorized into two 
classes: one that focuses on the users themselves handling such interruptions and the second focuses on enhancing or modifying aspects of system design in order to reduce or eliminate the interruptions' disruptive effects. Next, we briefly discuss each of these techniques with examples.

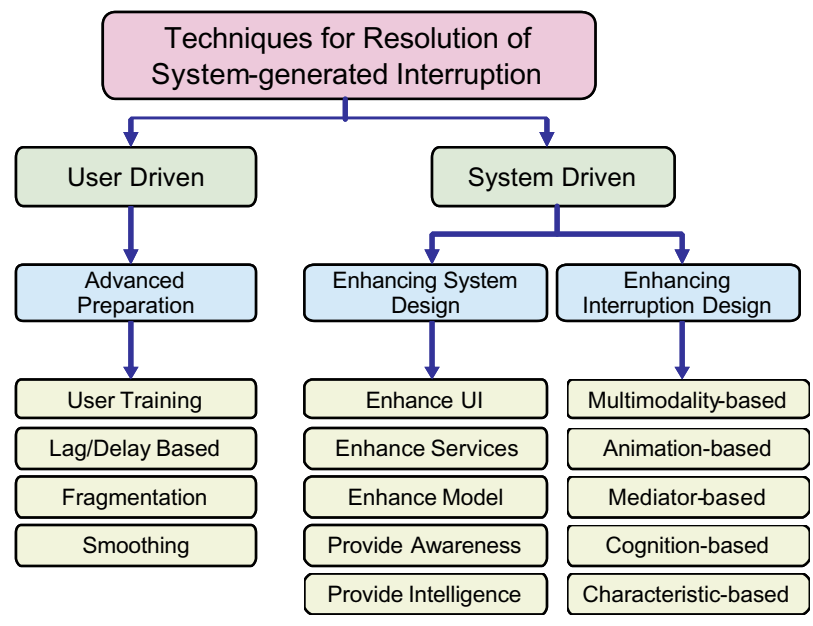

Figure 2. Taxonomy of Solutions for Handling System-generated Interruptions

\subsection{User Driven Approaches}

User driven approaches mainly focus on providing users with additional support, guidelines and information, in order to overcome interruptions without adversely affecting their task performance. Based on the relatively limited research in domains such as air traffic control, business environments, and so on, user driven approaches to interruption handling can be broadly categorized into four types, namely training-based, lag or delay based, fragmentation-based, and smoothing.

For training-based interruption solutions, research suggests that advanced training and preparation can be useful for handling interruptions in human computer interaction by employing rehearsal, memorization or context recovery for negating some effects caused by memory loss of the original task [18, 21, 40, 42]. Studies and experiments in prospective goal encoding and retrospective rehearsal $[11,22,47,54]$ indicate that users can employ interruption lag to prepare for their original tasks, suggesting that training and lag based methods work in combination to improve the performance after interruptions. Fragmentation of activities around interruption activities or vice versa also can provide users with some flexibility and reduce hindrances. Some studies and evaluations [7, 9, 35, 45] have been conducted which propose the division of tasks based on their phases of planning and execution such that interruptions can be handled more easily. Such studies also show that users' tasks are typically interleaved and it is possible to obtain specific points in multiple activities in order to interrupt the user for a least possible negative effect. On the other hand, research on smoothing by offering early warnings or cues indicates that benefit can be achieved if the interruptions are complex and time consuming, or socially disruptive as in cases of mobile devices [3, 16, 23]. Cues and warnings, either subtle or active, are important because they provide users with advance preparation time to more effectively handle interruptions.

These studies show that while user-driven methods can be employed as a first response towards handling interruptions, they do not necessarily produce significant increase in user performance or productivity and may also have the following drawbacks.

- Interruptions are not resolved, and the user herself has to take active steps to avoid hindrances caused by interruptions. This involves more time and cognitive effort spent in returning to the original tasks and results in performance improvements being marginal at best.

- Considerable increase in the overall workload of users. They have to perform pre-interruption and postinterruption activities to make interruptions smooth. These activities may involve memorizing or trying to remember the state of the original activity, making notes, preparing for impending interruptions, etc.

- Incur additional costs in terms of time, user training, capital and so on. This has been noticed especially in training activities for assembly lines, warehouses, etc. Not always do production managers have to apply scheduling or supply chain principles to avoid interruptions.

- Need to be effectively conducted. In case of multiple activities and frequent interruptions, users need to keep records of original tasks, state of each task, and variables involved before handling the interruptions so that they can smoothly switch back to those tasks after the interrupting tasks have been handled.

- Suited for applications where interruptions are routine, repetitive and predictive. In dynamic, mobile or responsive environments, the randomness of interruptions, situations and surrounding criteria have a bearing on these methods and make them less effective.

To summarize, user-driven approaches only partially resolve interruption handling, and involve considerable time and effort on the part of the user, neither of which is always possible or feasible. Below, we discuss the second category of interruptions handling techniques.

\subsection{System Driven Approaches}

In contrast to user-driven approaches, there is considerable amount of research in system driven approaches. This research however is distributed in a variety of disciplines such as workplace management, computer supported cooperative work (CSCW), user interface design, context-aware systems, artificial intelligence and so on. The majority of this research is also relatively limited in scope because it is application 
specific, or focuses on notifications and human computer interaction, rather than actual interruption handling. System driven approaches are broadly categorized into two types (Figure 2): one where system functionality or the underlying model is modified to resolve interruptions, while in the other interruptions themselves are enhanced to remove their disruptive properties. We discuss both of these categories below.

\subsubsection{Enhancing System Design}

The most popular and widely employed method of reducing hindrances caused due to interruptions has been to enhance the design of the system, its underlying model and its components, such as user interfaces, system services, and so on.

Research on enhancing system services such as Instant Messaging and communication [5, 13, 14] focuses on developing techniques for handling notifications. Evaluation of performance and responsiveness due to notifications conducted in such studies underscores the harmful effects of interruptions. Efforts on providing other services, for example those by Abowd et.al. and Ramchurn et.al., are based on user location to handle interruptions [1, 48]. Interruption handling here focuses on adapting services and interfaces based on user situation and location. Other areas of growing interest to interruption handling designers are those of smart homes and tangible interfaces. Due to responsiveness and dynamism in these types of systems, there is a necessity to effectively manage the services they provide. Surveys of research on smart homes [43] and interfaces [29] suggest that the use of learning techniques, sensors, ambient systems and wireless technologies has enabled such systems to become human centric, but as a result they have also become highly disruptive, partly due to their properties and partly due to lack of user training and experience in using such systems.

Interface design has been a widely targeted area for human computer interaction designers. Shneiderman [51] discusses user interface design as the first step towards achieving effective human computer interaction. It is also a way of resolving interruptions [6, 24, 31]. These works focus on developing innovative user interfaces that are dynamic and pervasive in nature and provide awareness of situations and events so that the user is prepared for eventual interruptions. Some of these are web-based, some are pervasive and located throughout the user's environment, while others are transparent and multilayered to provide users with the best possible means for maintaining task awareness.

In addition to user interface design and information awareness, enhancing the system model can also help in resolving interruptions and make interaction more human centric. Studies such as $[26,27,37,46]$ discuss the need for effective recognition of users' cognitive needs and limitations, attentional focus, decision making abilities and so on, during the design process in order to make user system interaction smooth and non-disruptive. Modeling languages and interface specification tools can be employed to develop system models to detect possible interruption scenarios.

Sensors, learning models, decision aids, information awareness bols, etc., can also help support systems in making interaction less disruptive. These can be used in conjunction with a variety of awareness, mining and learning techniques, to generate effective interruption aware system models. Such models allow systems to be aware of events, user behavior, surrounding situations and so on, thus allowing them to channel interruptions in a more appropriate manner. There is considerable research on providing awareness $[26,27,52,58]$ and intelligence $[19,20,25,26]$. Most of the focus here is on making systems dynamic, responsive and aware of users and their surroundings. This is an active approach wherein the system manages the interaction to suit the users' needs. Applications of these approaches include virtual office assistants, augmented instant messaging, virtual tour guides, cell phones, and so on.

\subsubsection{Enhancing Interruption Design}

Interruptions can also be resolved by enhancing the design of the interrupting entities themselves. This includes enhancing the interrupting modality, making the interruption mediator based, or animation based, making the interruptions cognitively simple and easy to resolve, and interrupting by changing the type of interruption modality.

Interrupting users based on the modality was studied by [4] and [12] where responses by the system to users during user-system interaction were enhanced by the use of various modalities such as heat, smell, sound, vibration and light. Results in these studies and others [56] indicate that active interruptions, such as sound, are more disruptive than passive interruptions, such as heat, light or vibration. In other words, differences in multimodal responses interrupt users to varying degrees. Other works employ similar multimodal techniques for user-system interaction to provide the user with appropriate interaction capabilities and reduce hindrance caused due to interruptions [36]. Animation or graphics based techniques [34, 38] are also being studied for passively interrupting users. While animation has not been solely used as the interruption modality, it is often used in combination with other GUI based methods seen in instant messaging, calendaring and scheduling software.

There is some research in the areas of mediator based, cognition based and characteristic based interruption handling. For instance, [5] and [15] employ agents-based software mediators that communicate between the users and the system responses to identify events and activities 
and provide smooth interruptions. Cognition based interruption handling involves the design of interruptions such that they can be easily understood and resolved by the user. This is usually done at the user interface level, and substantial literature is available on such design approach [46, 51]. Research on characteristic based interruption handling is rarer. For example [50] addresses different kinds of alarms for different types of interruptions in control room environments. In general, interrupting entities are handled manually (such as setting schedules, reminders, email notifications, cell phone tunes, and so on), while automated methods are based on high level user interface or system design requirements. Examples of these may be non-negotiable critical error messages, option driven dialog boxes, etc.

Research on system driven techniques suggests that they are more effective compared to user based solutions. However, it was noted that most system driven techniques are application specific and cannot be applied across domains. Many techniques do not address interruptions directly but rather as part of a higher set of requirements such as awareness or intelligence, and hence do not fully solve specific issues related to interruptions. It was also noticed that there is very little research, if any, on the development of frameworks and methodologies for handling interruptions, and there exists no comprehensive taxonomy to summarize the methods employed for interruption handling. In the next section we introduce and examine a proposed solution for interruption handling that incorporates a human perspective approach for developing interruption aware models.

\section{The Proposed Approach}

The proposed solution is a system driven approach, i.e., the system is designed in such a manner that it is able to learn about the situation a user may be in and interrupts appropriately such as to cause the least amount of hindrance. In order to do this, principles from human factors engineering, machine learning and software programming are employed. This approach not only improves system design but also enhances the interrupting components themselves.

The problem with most approaches that employ learning, recognition or awareness techniques for interruption handling is that they focus on human activity or behavior inference, which is an extremely complex task. This task is further complicated by the inherent uncertainty in human activity or behavior processes and their random dependence on surrounding conditions.

Instead, we aim to circumvent the complexity of activity inference by providing a simpler human perspective based approach that incorporates first hand information from users via surveys, field studies and experience sampling to accurately recognize and learn about the interruptability of a user. In doing so, uncertainty in activity or behavior inference is avoided while maintaining a high level of accuracy and scenario independence for interruption handling purposes.

Figure 3 illustrates our approach, which consists of a three-phase process, namely, pre-design information elicitation part, interruption-aware model learning phase, and a post-design preference elicitation part. The first part gathers information about user interruptability, day-to-day activity, responses, preferences, etc., by means of surveys, field studies and experience sampling. This is important because, it provides data for generating the learning model and also helps in selecting the attributes, sensors and other tools required for implementing such a system. The second part is where all the information gathered is converted into a trained learning model by employing machine learning algorithms. The conversion may be done via manual observation, statistical techniques, or automated mining tools. The conversion results in the generation of rules, policies, constraints and training datasets needed for building the learning model. The model can then be used to sense new events and situations and respond accordingly in an interruption aware manner.

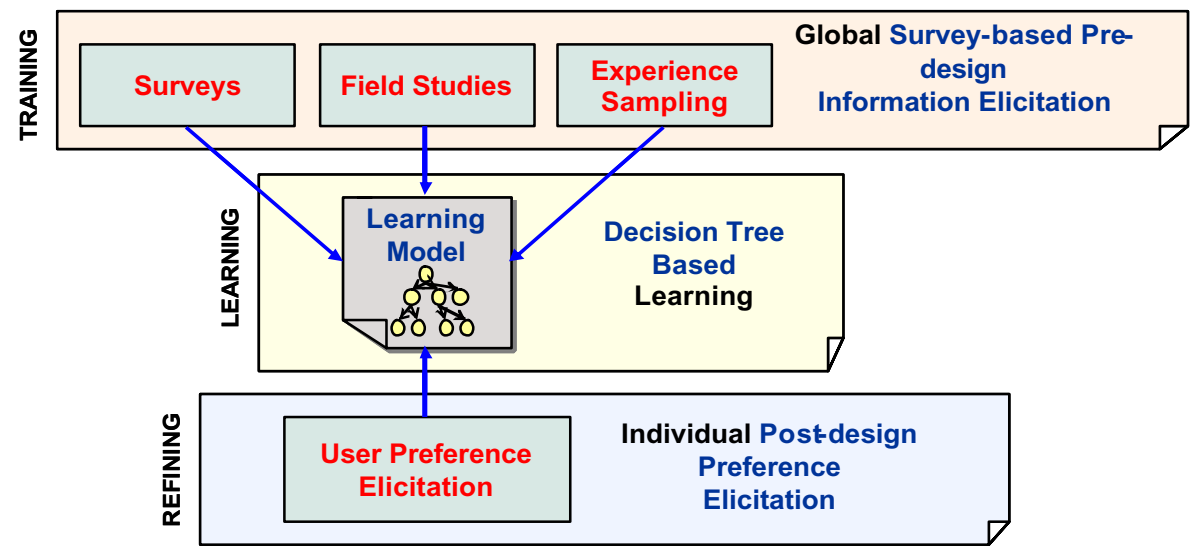

Figure 3. Proposed Approach Employing Pre- and Post- Design Elicitation for Learning Accurate Interruption-aware Models 


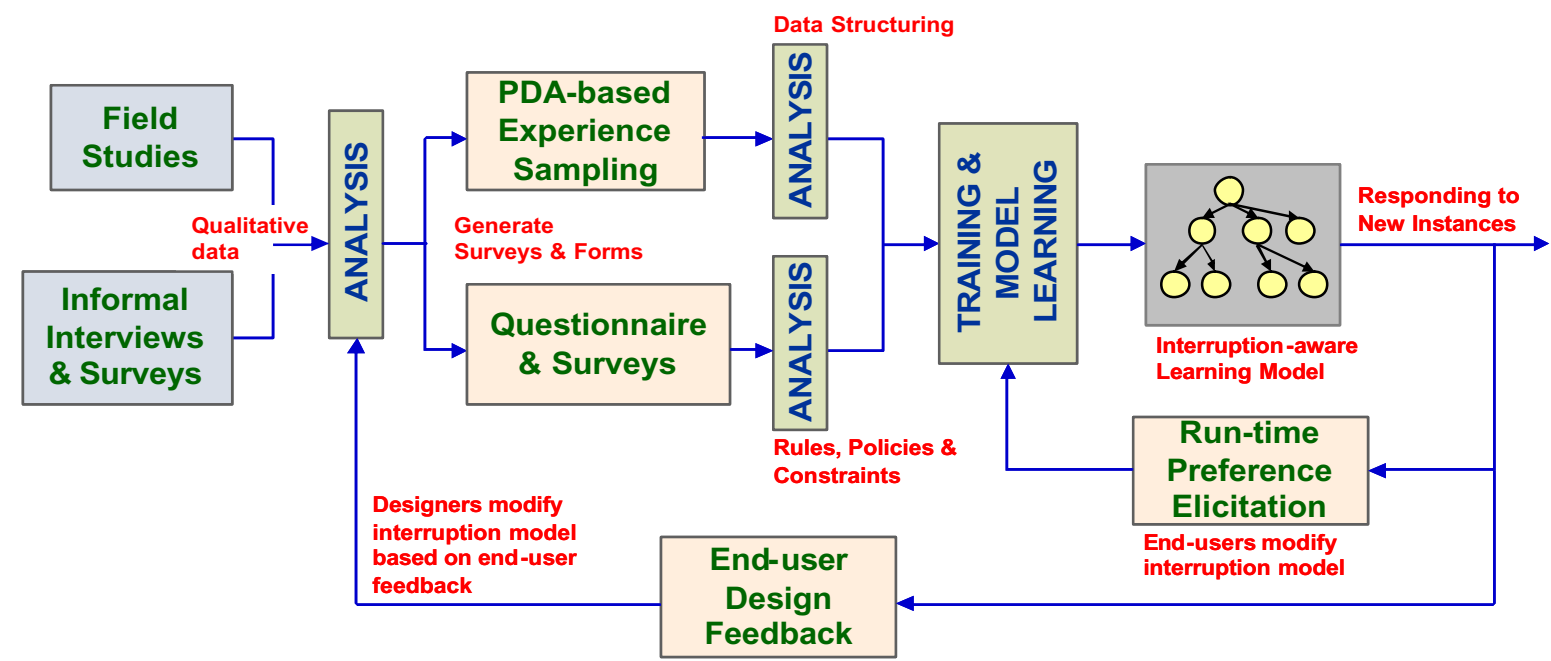

Figure 4. An Example: Employing Information Directly to Develop Interruption Aware Systems

But since user behavior and preferences are highly variable, there needs to be a mechanism that makes the system effective for any situation and any type of user. To resolve this, the third part is required which essentially gathers individual user's preferences during system use and refines the learned model appropriately. Machine learning approaches provide greater advantages than static rules or case based approaches .

Figure 4 shows an example of how the information elicitation process gathers data via the human perspective based approach, converts it to training information for the machine learning algorithms, which then generate respective interruption-aware models. These models can then be used to respond appropriately to new interruptions based on user situations. This human perspective based approach ensures that user preferences, needs, and other situational aspects are effectively incorporated. Work is currently being done in employing this approach to simulate diverse scenarios such as cell phones and virtual secretaries, both of which are highly prone to interruptions, and furthermore are experiencing rapid growth in popularity and use. Results of this work are not the scope of this paper but are reported in a forthcoming publication [59]. In the next section, we will see how our approach fits in a typical system design process.

\section{An Interruption Aware System Design Process}

Engineering design processes typically consist of a standard set of steps that need to be performed in sequence starting with activities like requirements analysis, goal determination, and ending with prototyping and evaluation [17, 44,57]. Many stages are repetitive because a developed entity may need to be modified and refined to suit design goals and requirements. Software design processes are similar to those for engineering design (e.g. Waterfall Model, or Spiral Model), with the main differences being the actual system design stages which may differ based on design approaches, application domains, enabling technologies, and so on [10].

Figure 5 shows a typical system design process, modified to incorporate human perspective based on information elicitation stages before and after the design has been completed. In a human perspective approach, such as that proposed here, the idea is to incorporate as much information as possible directly from end-users. As the Figure suggests, the first and last stages of the design process are similar to a conventional software design process. Prior to the actual design and implementation phases, scenario definitions, requirements analysis, goals, objectives and needs definitions, and feasibility studies are performed. For example, to design an interruption aware cell phone, designers need to formulate the scenario and its constraints, goals, use cases, environments, etc. They also need to determine its functional requirements, based on objectives, and needs from user studies. Feasibility studies are required to determine field usability, their projected technical needs, development costs, and any other relevant factors.

Once pre-design stages are complete, the methodology calls for pre-design information elicitation to gather data for use in the actual design process. This is done by employing a variety of user based data collection techniques like surveys, field studies and experience sampling. Currently, a manual data collection process is used in our simulations, but the idea is to employ user based design, participatory design, and so on, to automate the information elicitation process. This stage provides designers with knowledge about the selection of attributes, sensors, and other enabling technologies for implementation and prototyping stages. Other issues such as user preferences, characteristics, surroundings and interruptability are also determined here and incorporated into the system design. 


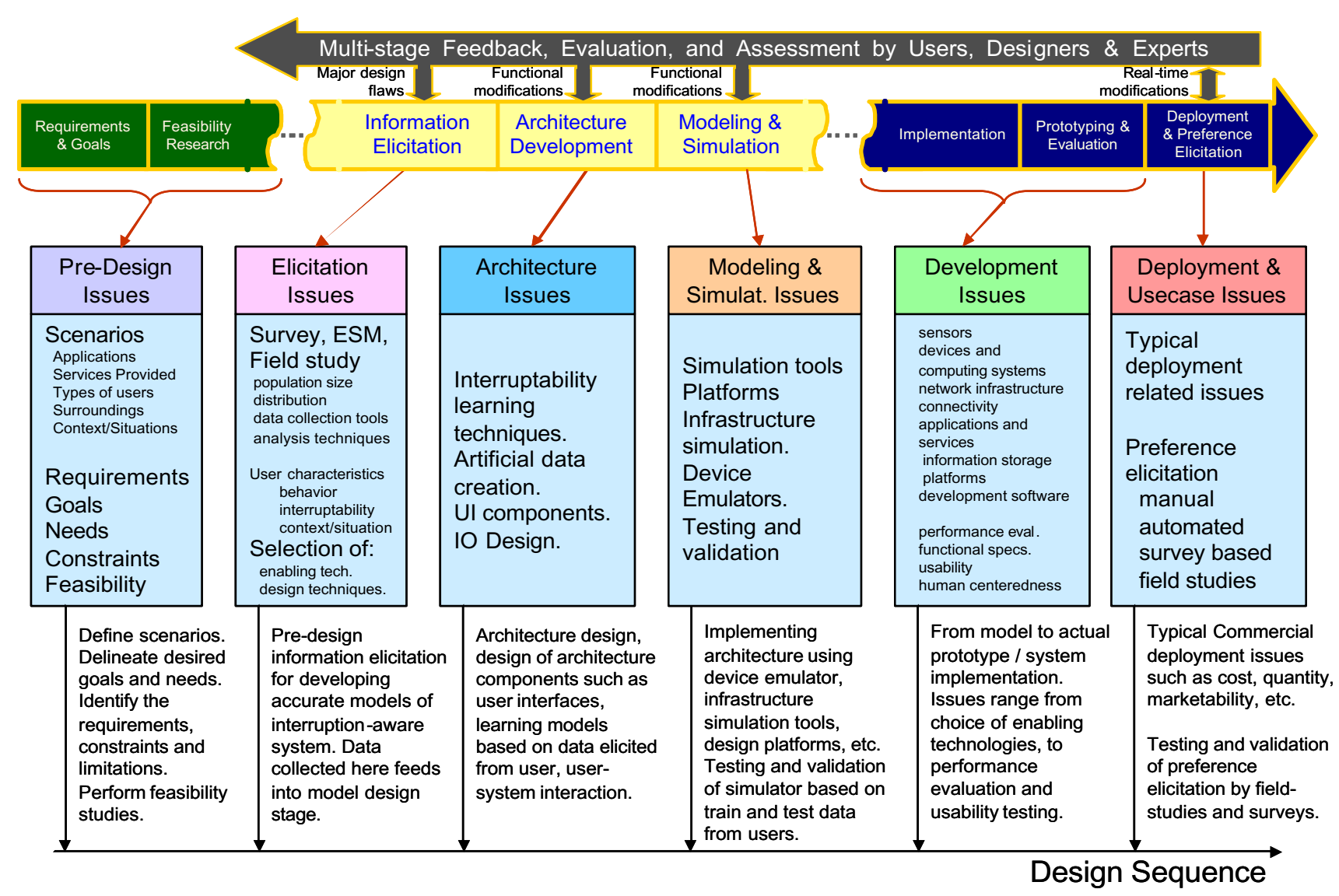

Figure 5. Methodology for the Design of Interruption-Aware Systems

Once this stage is complete, collected data can be used to develop rules, policies, constraints, and even training information for the development of models, and prototypes. After these are generated, they can be directly used in the implementation phase, or can be used to refine the architecture development, and sensor/component selection process. A host of enabling technologies can be employed, the choice of which normally would have been made during the initial design and elicitation stages. Prototypes developed are evaluated and tested, not only for functional performance but also usability, ergonomics, and other human and system factors such as userfriendliness, human centeredness, robustness, security, and so on.

Our methodology adds post-design preference elicitation activities in the development and deployment stages. In other words, after the interruption aware system has been deployed in the real world, users can perform preference based modifications to system functionality by supplying it with the required information directly. We are currently working on developing various scenarios using this methodology. Specifically, we are focusing on interruption aware cell phones and virtual gatekeepers. In this work, we employ our human perspective based approach to elicit information that is used to create training datasets for generating awareness models for both scenarios. The awareness models are then 'installed' in scenario simulators to test their functional and end-user validity. Further details of this work are currently being made in forthcoming papers.

\section{Conclusions and Future Work}

Given the current popularity of pervasive systems, intelligent devices, and human centric design approaches, what is required is a thorough understanding of how interruptions are caused and how they can be avoided. Better design principles and technologies can certainly help in reducing the effects of interruptions, while at the same time user driven approaches can offer temporary solutions for the problem. In this paper, we have addressed the subject of user interruptions generated by system events, notifications, errors and other activities. We have defined the problem, presented basic terminology, and discussed in depth the existing solutions available to address this problem. We have also discussed their advantages and shortcomings. In addition, we have tackled this problem with a solution that actively 
incorporates information from users and their surroundings in the design process, as well as during and after use. We also presented a general design process for human perspective based design of interruption aware systems. We have been implementing this design methodology and carrying out experimentation to verify the proposed ideas' performance and robustness. These results will be documented in future papers [59].

\section{References}

[1] Abowd, G.D., C.G. Atkeson, J. Hong, S. Long, R. Kooper, and M. Pinkerton, "Cyberguide: A Mobile Context-aware Tour Guide," Wireless Networks, Vol. 3, No. 5, pp. 421-433, Oct. 1997.

[2] Adamczyk, P.D., and B.P. Bailey, "If Not Now When? The Effects of Interruptions at Various Moments within Task Execution, In Human Factors and Computing Systems, Proceedings of CHI'04, pp. 271-278, 2004.

[3] Altmann, E.M., and J.G. Trafton, "Task Interruption: Resumption Lag and the Role of Cues," Proc. of the 26th Annual Conference of the Cognitive Science Society, Erlbaum Associates, pp. 42-47, 2004.

[4] Arroyo, E., T. Selker, and A. Stouffs, "Interruptions as Multimodal Outputs: Which are the Less Disruptive?" In Proceedings of the $4^{\text {th }}$ International Conference on Multimodal Interfaces, pp. 479-483, 2002.

[5] Avrahami D., and S.E. Hudson, "Balancing Performance and Responsiveness Using an Augmented Instant Messaging Client," Proc. of ACM Conference on Computer Supported Cooperative Work (CSCW'04), New York: ACM Press, 2004.

[6] Bailey, B.P., J.A. Konstan, and J.V. Carlis, "Adjusting Windows: Balancing Information Awareness with Intrusion," Proc. of HFWeb, 2000.

[7] Bailey, B.P., J.A. Konstan, and J.V. Carlis, "The Effects of Interruptions on Task Performance, Annoyance, and Anxiety in the User Interface," Proc. of INTERACT 2001, NCP, Tokyo, Japan, pp. 593-601, 2001.

[8] Bainbridge, L., "Diagnostic Skills in Process Operation," Proc. of the 1984 International Conference on Occupational Ergonomics, Vol. 2, pp. 1-10, 1984.

[9] Bannon L., A. Cypher, S. Greenspan, and M.L. Monty, "Evaluation and Analysis of Users' Activity Organization," In Proceedings of ACM CHI'83 Conference on Human Factors in Computing Systems, pp. 54-57, 1983.

[10] Behforooz, A., and F.J. Hudson, SOFTWARE ENGINEERING FUNDAMENTALS, Oxford Univ. Press, 1996

[11] Brandimonte, M., G.O., Einstein, and M.A. McDaniel, PROSPECTIVE MEMORY: THEORY AND APPLICATIONS. Erlbaum Associates, Mahwah, NJ, 1993.

[12] Brewster, S.A., P.C. Wright, and A.D.N. and Edwards, "The Design and Evaluation of an Auditory Enhanced Scrollbar," In Proceedings of the SIGCHI Conference on Human Factors in Computing Systems, pp. 173-179, 1994.

[13] Czerwinski, M., E. Cutrell, and E. Horvitz, "Instant Messaging and Interruption: Influence of Task Type on Performance," In Proceedings of the OZCHI Conference: Interfacing Reality in the New Millennium, pp. 356-361, 2000.
[14] Czerwinski, M.,E. Cutrell, and E. Horvitz, "Instant Messaging: Effects of Relevance and Time," In People and Computers XIV: Proceedings of HCI 2000, Vol. 2, pp. 71-76, 2000.

[15] Dekel, U., and S. Ross, "Eclipse as a Platform for Research on Interruption Management in Software Development," Eclipse Technology Exchange Workshop at OOPSLA'04, Vancouver, BC, Canada, pp. 12-16, October 2004.

[16] Detweiler, M.C., S.M. Hess, and M.P. Phelps, "Interruptions and Working Memory," Unpublished Technical Report, The Pennsylvania State University, Department of Psychology.

[17] Ertas, A., and J.C. Jones, THE ENGINEERING DESIGN PROCESS, John Wiley and Sons Inc., 1992.

[18] Franke, J., J. Daniels, and D. McFarlane, "Recovering Context After Interruption," Proc. of the 24th Annual Meeting of the Cognitive Science Society, Fairfax, VA, Aug. 8-10, pp. 310-315, 2002

[19] Gibbs W.W., “Considerate Computing," Scientific American, Vol. 292, No. 1, pp. 54-61, 2005.

[20] Gievska S., and J. Sibert, "Using Task Context Variables for Selecting the Best Timing for Interrupting Users," Proc. of the 2005 Joint Conference on Smart Objects and Ambient Intelligence: Innovative Context-Aware Services: Usages and Technologies, New York: ACM Press, pp. 171-176, 2005.

[21] Gillie, T., and D.E. Broadbent, "What Make Interruptions Disruptive? A Study of Length, Similarity, and Complexity," Psychological Research, Vol. 50, pp. 243-250, 1989.

[22] Goschke, T., J. Kuhl, "Representation of Intentions: Persisting Activation in Memory," J. of Experimental Psychology: Learning, Memory, \& Cognition, Vol. 19, No. 5, pp. 1211-1226, 1993.

[23] Hansson, R., P. Ljungstrand, and J. Redstrom, "Subtle and Public Notification Cues for Mobile Devices," LNCS, UBICOMP, Vol. 22, No. 1, pp. 240-247, 2001.

[24] Harrison, B.L., H. Ishii, K.J. Vincente, and W.A.S. Buxton, "Transparent Layered User Interfaces: An Evaluation of a Display Design to Enhance Focused and Divided Attention," Proc. of Human Factors in Computing Systems Conference (CHI '95), pp. 317-324, 1995.

[25] Horvitz E., A. Jacobs, and D. Hovel, "Attention Sensitive Alerting," Proc. of the 15th Conference on Uncertainty and Artificial Intelligence (UAI'99), San Francisco: Morgan Kaufmann, pp. 305-313, 1999.

[26] Horvitz, E., and J. Apacible, "Learning and Reasoning about Interruption," Proc. of the ACM International Conference on Multimodal Interfaces, pp. 20-27, 2003.

[27] Horvitz, E., C. Kadie, T. Paek, and D. Hovel, "Models of Attention in Computing and Communication: From Principles to Applications," Comm. of the ACM, Vol. 46, No. 3, pp. 52-59, 2003.

[28] Hudson S., J. Fogarty, C. Atkeson, D. Avrahami, J. Forlizzi, S. Kiesler, J. Lee, and J. Yang, "Predicting Human Interruptability with Sensors: A Wizard of $\mathrm{Oz}$ Feasibility Study," Proc. of the ACM Conference on Human Factors in Computing Systems (CHI'03), New York: ACM Press, pp. 257264, 2003. 
[29] Ishii, H., B. Ullmer, "Tangible Bits: Towards Seamless Interfaces between People, Bits and Atoms," Proc. of the ACM Conference on Human Factors in Computing systems (CHI 97), Atlanta, Georgia, March, pp. 231-241, 1997.

[30] Latorella, K.A., "Investigating Interruptions: Implications for Flight Deck Performance," NASA Technical Memorandum 209707, National Aviation \& Space Administration, Washington, DC, 1999.

[31] Lee, J.C., "Spatial User Interfaces: Augmenting Human Sensibilities in a Domestic Kitchen," Master Thesis, School of Architecture and Planning, MIT Media Laboratory, 2005.

[32] Liu, K.K. "A Personal, Mobile System for Understanding Stress and Interruptions," S.M. Thesis, Massachusetts Institute of Technology, 2004.

[33] Madhaven, D., and K. Funk, "Cockpit Task Management Errors in Critical In-flight Incidents," Proc. of the 7th Intern. Symposium on Aviation Psychology, pp. 970-974, 1993.

[34] Maes, P., "Agents That Reduce Work and Information Overload,” CACM, Vol. 37, No. 7, pp. 30-40, July 1994.

[35] Mark, G., V.M. Gonzalez, J. Harris, "No Task Left Behind? Examining the Nature of Fragmented Work," Proc. of the ACM Conference on Human Factors in Computing systems CHI: Interruptions and Attention 1, Portland, Oregon, pp. 321-330, April, 2005.

[36] MoGee, D.R., and P. Cohen, "Exploring Handheld, Agentbased, Multimodal Collaboration," Proc. of the Workshop on Handheld Collaboration, Conference on Computer Supported Cooperative Work (CSCW), Seattle, WA,1998.

[37] McCrickard, D.S., C.M. Chewar, "Attuning Notification Design to User Goals and Attention Costs," Comm. of the ACM, Vol. 46, No. 3, pp. 67-72, March 2003.

[38] McCrickard, D.S., R. Catrambone, and J.T. Stasko, "Evaluating Animation in the Periphery as a Mechanism for Maintaining Awareness," Proc. of INTERACT 2001, Tokyo, Japan, pp. 148-156, July 2001.

[39] McFarlane, D.C., "Coordinating the Interruption of People in Human-Computer Interaction,” Proc. of INTERACT'99, IOS Press, pp. 295-303, 1999.

[40] McFarlane, D.C., "Comparison of Four Primary Methods for Coordinating the Interruption of People in HumanComputer Interaction," Proc. of HCI'02, Vol. 17, pp. 63-139, 2002.

[41] McFarlane, D.C., "Interruption of People in HumanComputer Interaction: A General Unifying Definition of Human Interruption and Taxonomy," NRL Formal Report, NRL/FR/5510 - 97-9870, Washington, DC, Naval Research Laboratory, 1997.

[42] McFarlane, D.C., and Latorella, K. A., "The Scope and Importance of Human Interruption in HCI Design," HumanComputer Interaction, Vol. 17, pp. 1-61, 2002.

[43] Meyer, S., and A. Rakotonirainy, "A Survey of Research on Context-aware Homes," Conf. on Research and Practice in Information Technology Series, Proc. of the Australasian Information Security Workshop, Conference on ACSW Frontiers, Vol. 21, Adelaide, Australia, pp 159-168, 2003.

[44] Middendorf, W.H., DESIGN OF DEVICES AND SYSTEMS, $2^{\text {nd }}$ Edition, Marcel Dekker Inc., 1989.
[45] Monk, C.A., D.A. Bohem-Davis, and J.G. Trafton, "The Attentional Costs of Interrupting Task Performance at Various Stages," Proc. of the 46th Annual Meeting of the Human Factors and Ergonomics Society, Santa Monica, pp. 1824-1828, 2002.

[46] Norman, D.A., COGNITIVE ENGINEERING: USER CENTERED SYSTEM DESIGN, D.A. Norman and S.W. Draper, Editors, Lawrence Erlbaum, Hillsdale, NJ, 1986.

[47] Patalano, A.L., C.M. Seifert, "Opportunistic Planning: Being Reminded of Pending Goals," Cognitive Psychology, Vol. 34, pp. 1-36, 1997.

[48] Ramchurn, S.D., B. Deitch, M.K. Thompson, D.C. DeRoure, N.R. Jennings, and M. Luck, "Minimising Intrusiveness in Pervasive Computing Environments Using Multi-agent Negotiation," Proc. of the First International Conference on Mobile and Ubiquitous Systems , Boston, MA, pp. 364-372, 2004.

[49] Rouncefield, M., J.A. Hughes, T. Rodden, and S. Viller, "Working with Constant Interruption: CSCW and the Small Office," Proc. of the CSCW '94 Conf. on Computer-Supported Cooperative Work, New York, NY, pp. 275-286, 1994.

[50] Savioja, P., "Alarms - Necessary Interruptions?," T.121900 Seminar on User Interfaces and Usability, HUT, Sober IT, 2004.

[51] Shneiderman, B., DESIGNING THE USER INTERFACE: STRATEGIES FOR EFFECTIVE HUMAN-COMPUTER INTERACTION, 3rd Ed., Addison-Wesley, Reading, MA, 1997. [52] Siewiorek D., A. Smailagic, J. Furukawa, A. Krause, N. Moraveji, and K. Reiger, "SenSay: A Context-aware Mobile Phone," Proc. of the IEEE Intern. Symposium on Wearable Computers, New York, October, pp. 248-249, 2003.

[53] Speier, C., I. Vessey, and J.S. Valacich, "The Effects of Interruptions, Task Complexity, and Information Presentation on Computer-Supported Decision-Making Performance," Decision Sciences, Vol. 34, pp. 771-, 2003.

[54] Trafton, J. G., E.M. Altmann, D.P. Brock, and F.E., "Preparing to Resume an Interrupted Task: Effects of Prospective Goal Encoding and Retrospective Rehearsal," Intern. Journal of Human-Computer Studies, Vol. 58, pp. 583-603, 2003.

[55] Van Solingen, R.,E. Berghout, F. Van Latum, "Interrupts: Just A Minute Never Is," IEEE Software, Vol.15, No. 5, pp. 97103, 1998.

[56] Welch, R.B., and D.H. Warren, "Intersensory Interactions," Handbook of Perception and Human Performance, Chapter 25, New York: Wiley, 1986.

[57] White, J.R., and T.L. Booth, "Towards an Engineering Approach to Software Design," Proc. of the $2^{\text {dd }}$ International Conference on Software Engineering, San Francisco, California, October, pp. 214-222, 1976.

[58] Yan H., and T. Selker, "A Context-Aware Office Assistant," ACM International Conference on Intelligent User Interfaces, New Orleans, LA, January, pp. 276-279, 2000.

[59] Godbole, A. and W.W. Smari, "Experiences in Acquisition, Learning and Awareness based Design and Implementation of a Context-Aware System," SIMA, the 2006 Workshop on Awareness, MILCOM 2006, Washington, D.C., October 23-25, 2006, Accepted. 